\title{
Advanced Testing and Modeling of Magnetic Materials Including a New Method of Core Loss Separation for Electrical Machines
}

\author{
Maged Ibrahim, Student Member, IEEE, Pragasen Pillay, FIEEE
}

\begin{abstract}
This paper presents a new method for the separation of core loss components (hysteresis and eddy current) in laminations exposed to high frequency excitations. Accurate separation of core losses is achieved by calculating the hysteresis losses at each frequency taking into account the non-uniform flux distribution inside the lamination. The results highlight that the assumption of constant hysteresis energy loss per cycle is only valid at low frequencies, where skin effect is negligible. The developed model is then used to study the effect of the annealing process on core loss components in laminations exposed to high frequency excitations. Core loss measurements are performed on different laminations at several frequencies in the range of $20 \mathrm{~Hz}$ $-4000 \mathrm{~Hz}$. A comparison of the separated core loss components shows that a huge reduction in the hysteresis losses is achieved by annealing, while the annealing process increases the eddy current loss component at high frequencies and high flux densities. The results are then analyzed by comparing the separated eddy current loss with an analytical eddy current loss model that accounts for the non-uniform distribution of the magnetic field.
\end{abstract}

Index Terms - Core loss, Annealing, Eddy current loss, Hysteresis loss, Skin effect.

\section{INTRODUCTION}

$\mathrm{C}$ Yore losses in electrical machines account for a significant portion of the total losses ranging from $15-25 \%$ in machines operating with $50 / 60 \mathrm{~Hz}$ sinusoidal supplies [1] and even higher for newer high speed designs. The introduction of variable speed drives (VSDs) increases the demand on high speed electrical machines. These are appealing choices for certain applications, such as aerospace generators and flywheels, as increasing the machine speed allows a reduction in the machine size and weight for the same output power. However, in high speed applications core loss increases, and it becomes a limiting electrical constraint along with the mechanical constraints of vibrations and bearings. Furthermore, in conventional induction motors used in industry, core losses increase when the motor is driven by VSDs, as the flux waveform inside the motor laminations

Manuscript received October 19, 2011.

M. Ibrahim is with the Department of Electrical and Computer Engineering, Concordia University, Montreal, QC, Canada, (e-mail: ma_ib@encs.concordia.ca).

P. Pillay is with the Department of Electrical and Computer Engineering, Concordia University, Montreal, QC, Canada, and also an adjunct Professor at the University of Cape Town, Cape Town, South Africa (e-mail: pillay@encs.concordia.ca). contains high harmonic content that is produced by the VSDs distorted voltage waveforms.

The design of modern electrical machines requires accurate quantification of core losses over a wide frequency and flux density range. Various core loss models have been developed to estimate core losses. Most of the early work was done by Steinmetz [2]. In his work, core losses are considered as the addition of hysteresis and eddy current loss, and the total core loss is given by,

$P=K_{h} f B^{n}+K_{e} f^{2} B^{2}$

$K_{h}, K_{e}$ and $n$ are the loss coefficients which are dependent on the material. The two-term formulation shows large discrepancies compared to the experimental results, especially at high flux densities and high frequencies. This difference is normally explained by the excess losses. Bertotti [3] proposed an additional term to explain these excess losses based on the statistical loss theory, and the total core loss is expressed as,

$P=K_{h} f B^{n}+K_{e} f^{2} B^{2}+K_{e x} f^{1.5} B^{1.5}$

where $K_{e x}$ is dependent on the material micro-structure, the conductivity, and the cross section area of the lamination. The coefficients of the three-term formulation are generally obtained from the measured core loss data. However, the calculated losses from (2) are only accurate within a certain frequency and flux density range. In more recently developed models [4, 5], this range is extended by allowing the coefficients to vary with the frequency and the flux density. However, the determination of these variable coefficients requires additional core loss data at high frequencies and high flux densities.

Recently, one of the authors [6] demonstrated, by means of finite element (FE) simulation, that the excess losses are mainly attributed to the non-uniform distribution of the magnetic field inside the lamination created by both the skin effect and the non-linear diffusion of the magnetic field. Therefore, the total core losses can be represented by the hysteresis and eddy current losses considering the realistic field distribution.

The effect of the non-uniform magnetic field distribution on 
the lamination losses has been extensively studied in the literature $[6,7,15,16,19$, and 20]. While many analytical and numerical models have been developed to calculate the eddy current losses considering skin effect, there is no study conducted on the variation of the hysteresis energy loss due to the non-uniform magnetic field distribution. This variation does not only affect the prediction of the hysteresis loss component at high frequency, but it also changes the experimentally separated eddy current losses.

Section II reviews the methods of core loss separation. Section III presents a new method of core loss separation considering the influence of the non-uniform flux distribution on the hysteresis losses. Section IV investigates the effect of annealing on core loss components in laminations exposed to high frequency excitations. Section V concludes the paper.

\section{REVIEW OF CORE LOSS SEPARATION METHODS}

Ideally, the static hysteresis energy loss per cycle is calculated by measuring the area enclosed by the hysteresis loop under static excitation. However, the measurement of the static loop requires special instrumentation [8]. When the magnetic material is exposed to a time varying magnetic field, the area enclosed by the measured hysteresis loop represents the total core loss per cycle, which includes both the hysteresis and eddy current loss components. Therefore, an alternative method is usually used to calculate the hysteresis energy loss per cycle using core loss measurements at different frequencies. In this method, the hysteresis loss per cycle is separated by extrapolating core loss per cycle vs. frequency curves at different flux densities to zero frequency. The power loss at zero frequency represents the hysteresis energy loss per cycle. Fig. 1 illustrates the separation of core loss components by the extrapolation method.

In the extrapolation method, the hysteresis energy loss per cycle is assumed independent of the frequency, and the total hysteresis loss is simply calculated by multiplying the static hysteresis energy loss per cycle by the operating frequency. This assumption is only valid at low frequencies, where the magnetic field across the lamination is uniform. When the lamination is exposed to a time-varying magnetic field, eddy currents are generated inside the lamination. These eddy currents, according to Lenz's law, produce a secondary magnetic field that opposes the applied field. The eddy current magnetic field is maximum at the centre of the lamination, where the contribution of all eddy currents adds, and minimum at the lamination surface. The actual magnetic field inside the lamination is the summation of the applied field and the eddy current field. Therefore, the distribution of the actual magnetic field is non-uniform across the lamination thickness, being maximum at the surface and minimum at the centre of the lamination. The confinement of the magnetic field around the lamination surface is known as skin effect [9]. At low frequencies, the generated eddy currents are small, and skin effect can be neglected. At high frequencies, skin effect is significant, and the peak flux density differs across the lamination thickness. As a result, the local hysteresis loop and the hysteresis energy loss per cycle varies at each point inside the lamination. This variation affects the total hysteresis

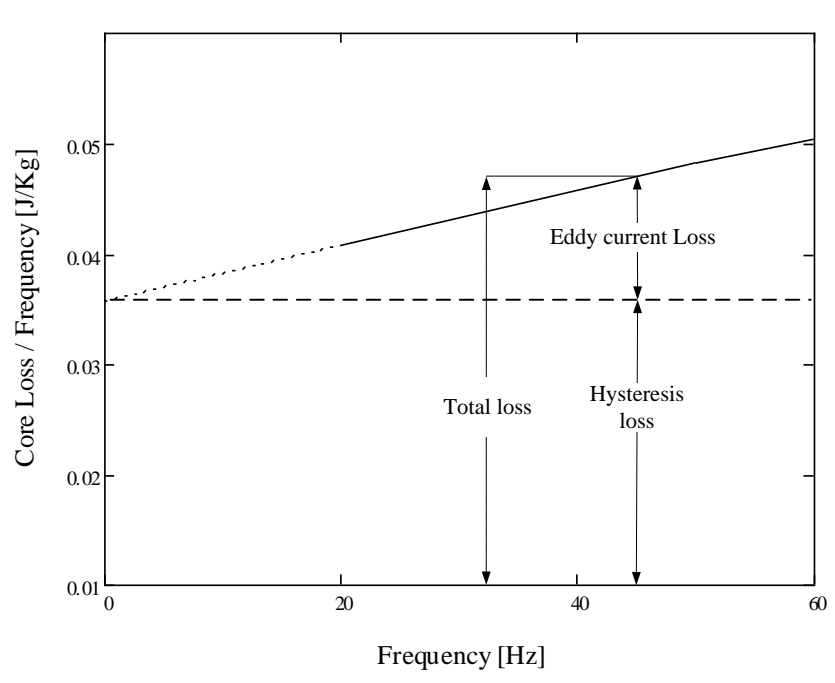

Fig. 1. Separation of core loss by the extrapolation method.

energy loss per cycle, making it dependent on the magnetic field distribution, which is strongly affected by the frequency. Therefore, the separation of core loss components by the extrapolation method, assuming constant hysteresis energy loss per cycle, is only valid at low frequencies. When skin effect is significant at high frequencies, accurate core loss separation requires taking skin effect into account in the determination of core loss components.

To achieve an accurate core loss separation, a dynamic finite element model for the non-linear hysteresis loop is developed by one of the authors [10]. In the model, Maxwell's Equations are solved using the finite element method, where the hysteresis at any location of the material is represented by an energetic hysteresis model [11]. The total losses are assumed to be composed of eddy current and hysteresis losses, which are calculated from the following equations,

$$
\begin{aligned}
& P_{e}=\frac{1}{\rho \iint d t d v} \iint d v d t \frac{\vec{J}_{e}^{2}}{\sigma} \\
& P_{h}=\frac{1}{\rho \iint d t d v} \iint d v \cdot \vec{H} d \vec{B}
\end{aligned}
$$

where $P_{e}$ is the eddy current loss in unit mass and unit time, $P_{h}$ is the hysteresis loss in unit mass and unit time, $\rho$ is the mass density of the magnetic material,$\vec{J}_{e}$ is the eddy current density in the lamination, $\sigma$ is the electric conductivity, $\vec{B}$ is the magnetic flux density, and $\vec{H}$ is the magnetic field intensity. The eddy current and hysteresis losses calculated in the finite element simulation considering the realistic nonuniform magnetic field are able to predict the measured core losses for frequencies up to $4000 \mathrm{~Hz}$.

Table I compares the hysteresis losses calculated by the FE model and the extrapolation method for three different materials. It can be seen that at $50 \mathrm{~Hz}$ the hysteresis losses obtained from the two methods are in good agreement. While at $1000 \mathrm{~Hz}$, the extrapolation method shows large errors in the 
TABLE I

FE MODFI. Vs. EXTRAPOI.ATION

\begin{tabular}{|c|c|c|c|c|}
\hline Material & $\begin{array}{c}\text { Frequency } \\
{[\mathrm{Hz}]}\end{array}$ & $\begin{array}{c}\text { Extrapolation } \\
\text { Hysteresis Loss } \\
\text { [W/Kg]* }\end{array}$ & $\begin{array}{c}\text { FE Hysteresis } \\
\text { Loss } \\
{[\mathrm{W} / \mathrm{Kg}]^{*}}\end{array}$ & $\begin{array}{c}\% \\
\text { Error** }\end{array}$ \\
\hline \multirow{2}{*}{ M19 G29 } & 50 & 0.77 & 0.80 & 3.75 \\
\hline & 1000 & 15.37 & 20.53 & 25.13 \\
\hline \multirow{2}{*}{$\begin{array}{c}\text { Mill A } \\
\text { annealed }\end{array}$} & 50 & 1.06 & 1.06 & -0.21 \\
\hline & 1000 & 21.23 & 32.30 & 34.27 \\
\hline \multirow{2}{*}{$\begin{array}{l}\text { Mill A un- } \\
\text { annealed }\end{array}$} & 50 & 6.02 & 6.03 & 0.18 \\
\hline & 1000 & 120.33 & 136.99 & 12.16 \\
\hline $\begin{array}{l}* \text { At a flux d } \\
* * \text { Based on }\end{array}$ & $\begin{array}{l}\text { ity of } 1 \mathrm{~T} \\
\text { respective }\end{array}$ & E results & & \\
\hline
\end{tabular}

uniform across the lamination, and the impact of skin effect on the hysteresis losses can be neglected. While at higher frequencies, skin effect is significant. This causes a deviation between the actual and the static hysteresis energy loss per cycle at high frequency. Therefore, it is only justified to use extrapolation at low frequencies.

Accurate separation of core loss components can be achieved by the FE model with relatively short computing time compared to other FE models. However, it is still impractical to use for establishing an extensive database of core loss components for various materials at different frequencies and flux densities. Therefore, an alternative method needs to be developed.

\section{FINITE SECTION METHOD FOR CORE LOSS SEPARATION}

This section presents a fast and efficient method for the separation of core losses at high frequencies using an analytical model to describe the flux density distribution across the lamination.

\section{A. Determination of the static hysteresis loss}

The static hysteresis energy loss per cycle is obtained from the measured core losses at two frequencies. These frequencies are chosen so that the influence of skin effect on the core loss components is negligible. When skin effect is negligible, the hysteresis energy loss per cycle is independent of frequency and the total hysteresis losses can be obtained by multiplying the frequency by the static hysteresis energy loss per cycle. Also, the eddy current loss can be assumed dependent on the square of the frequency. Thus, the total core loss $P_{t}$ can be simply given by,

$P_{t}=K f^{2}+W_{h} f$

where $K$ depends on the considered lamination and the flux density, and $W_{h}$ is the hysteresis energy loss per cycle. Using core loss data $P_{t 1}$ and $P_{t 2}$ at frequencies $f_{1}$ and $f_{2}, W_{h}$ can be calculated from,

$$
W_{h}=\frac{\left(P_{t 2} f_{1}^{2}\right)-\left(P_{t 1} f_{2}^{2}\right)}{\left(f_{1} f_{2}\right)\left(f_{1}-f_{2}\right)}
$$

The hysteresis loss formula in (1) with constant flux density exponent is found to be applicable for flux densities lower than $1 \mathrm{~T}$, which is less than the flux density level in most electrical machines. In order to extend it to higher flux densities, the formula was modified in $[4,12]$ to,

$W_{h}(B)=K_{h} B^{a+b B+c B^{2}}$

where $K_{h}, a, b$ and $c$ are curve fitting constants obtained from the separated hysteresis energy loss per cycle by (6).

\section{B. Distribution of Flux Density}

In order to study the influence of skin effect on high frequency hysteresis losses, a flux distribution model is adopted. The flux density amplitude at a given position $y$ from the center of the lamination is given in [8] as,

$B_{p}(\hat{y})=B_{b} \sqrt{\frac{\cosh \left(\frac{2 \hat{y}}{\lambda}\right)+\cos \left(\frac{2 \hat{y}}{\lambda}\right)}{\cosh \left(\frac{2}{\lambda}\right)+\cos \left(\frac{2}{\lambda}\right)}}$

$\lambda=\sqrt{\frac{2}{L^{2} \omega \mu \sigma}}$,

where $\hat{y}=y / L . B_{b}, \omega, \mu, L$ and $\sigma$ are the flux density amplitude at the boundary, the angular frequency, the magnetic permeability, the mass density, half the lamination thickness and the electrical conductivity, respectively.

The measured parameter in the experiment is the average flux density $B_{\text {ave }}$ which deviates from the flux density at the boundary, when skin effect is present. The peak average flux density is given in [7] by,

$$
B_{\text {ave }_{p}}=B_{b} \lambda \frac{\sqrt{0.5\left[1+\exp \left(\frac{8}{\lambda}\right)\right]-\exp \left(\frac{4}{\lambda}\right) \cos \left(\frac{4}{\lambda}\right)}}{1+2 \cos \left(\frac{2}{\lambda}\right) \exp \left(\frac{2}{\lambda}\right)+\exp \left(\frac{4}{\lambda}\right)}
$$

(8) and (10) are obtained from the solution of Maxwell's equation with an assumption of a linear magnetic material. However, the distribution of flux density is dependent on the magnetic permeability which varies along the hysteresis curve depending on the flux density level. The variation of magnetic permeability affects the distribution of flux density across the lamination thickness, being non-uniform for high values of magnetic permeability and more uniform for low values of permeability at low fields and near saturation. To account for the variation of field distribution with the flux density level, the magnetic permeability is allowed to vary with the flux 
density. Therefore, the permeability is expressed as a 4th order polynomial,

$$
\mu(B)=K_{0}+K_{1} B+K_{2} B^{2}+K_{3} B^{3}+K_{4} B^{4}
$$

where $K_{0}$ to $K_{4}$ are curve fitting coefficients obtained from the measured magnetic permeability at low frequency. Using (8-11), the distribution of flux density across the lamination at a certain measured average flux density can be calculated, knowing the operating frequency and the properties of the lamination.

In order to validate the analytical flux model, its results are compared with the flux waveforms obtained from the FE model. The results, in Fig. 2, show good correlation between the flux waveforms obtained from the two models at different locations inside the lamination. This means that the analytical flux model can calculate accurately the peak flux density variation across the lamination thickness, and therefore can be used to determine the local hysteresis loops.

When skin effect is significant, the value of the measured average flux density is no longer an accurate measure of the actual flux density inside the lamination which differs in magnitude at each point across the lamination thickness. In addition to the magnitude variation caused by skin effect, there is also a phase difference in the flux density waveforms at different points across the lamination. This time delay causes the peak flux density at different points to occur at different instants of time, and therefore the measured average flux density is not necessarily the average value of these peak flux densities. For all the above mentioned cases, the actual hysteresis energy loss per cycle at high frequency is no longer equal to the static hysteresis energy loss per cycle at a certain measured average flux density. Therefore, accurate quantification of hysteresis losses at high frequency requires considering the realistic flux distribution inside the lamination.

\section{Finite section method}

A model is implemented to achieve accurate quantification of high frequency hysteresis losses, taking into account the realistic flux distribution. The calculation starts with constructing the flux density distribution across the lamination thickness using (8-11). The lamination is then divided into $\mathrm{n}$ sections. At each section, the hysteresis energy loss per cycle is calculated from (7), depending on the value of the peak flux density. The total hysteresis loss is then calculated by summing the power loss of all the sections. Fig. 3 illustrates the variation of hysteresis loops across the lamination thickness. A flow chart of the model is shown in Fig. 4. The model results, displayed in Fig. 5, show that the hysteresis energy loss per cycle increases at high frequencies, especially when skin effect is significant at high values of magnetic permeability. While at low fields and near saturation, the hysteresis energy loss per cycle is almost equal to its values at low frequencies. This variation affects the hysteresis loss curve, making it more linear at high frequencies. This implies that the flux density exponent in the original Steinmetz equation should be a function of the frequency, as well as the flux density.
The model results are compared with the hysteresis losses calculated by the FE model and the extrapolation method. Table II compares the hysteresis losses obtained by the three methods at different frequencies at $1 \mathrm{~T}$ and $1.5 \mathrm{~T}$ for unannealed laminations. It can be seen that the model results are in good agreement with the FE hysteresis losses at all the tested frequencies up to $2000 \mathrm{~Hz}$. In addition, at low frequency, when skin effect is negligible, the extrapolation method shows good accuracy compared to the model and FE results. At high frequency, the hysteresis losses calculated the extrapolation method shows large errors up to $17.65 \%$ at $2000 \mathrm{~Hz}$ and $1 \mathrm{~T}$ compared to the model results. Also, it is clear from table II that the variation of the hysteresis energy loss per cycle with frequency is more severe at $1 \mathrm{~T}$ than at $1.5 \mathrm{~T}$ where the magnetic field distribution is more uniform.

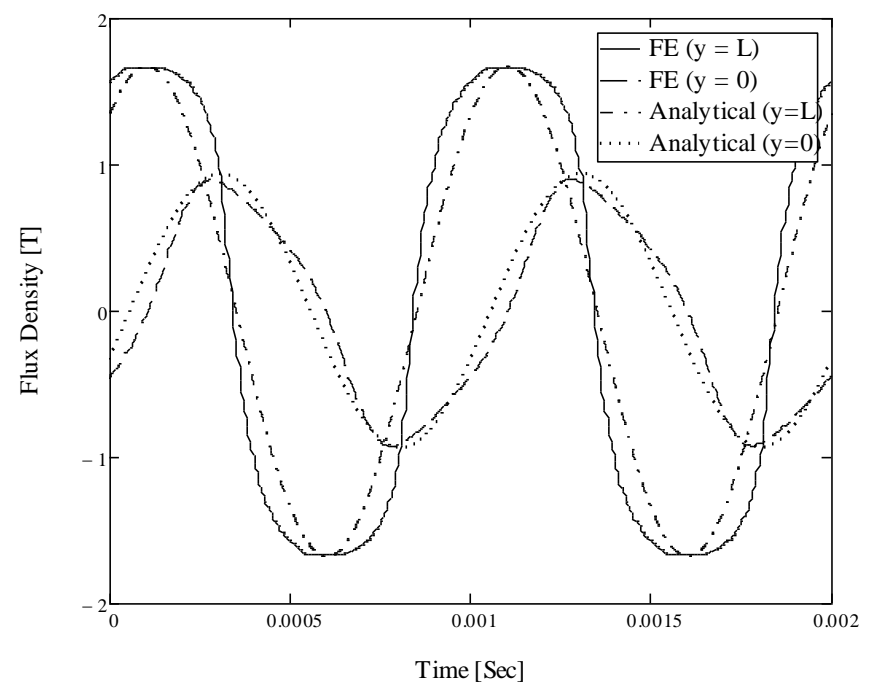

Fig. 2. Comparison of FE and analytical flux waveforms at different locations for un-annealed laminations at $1000 \mathrm{~Hz}$

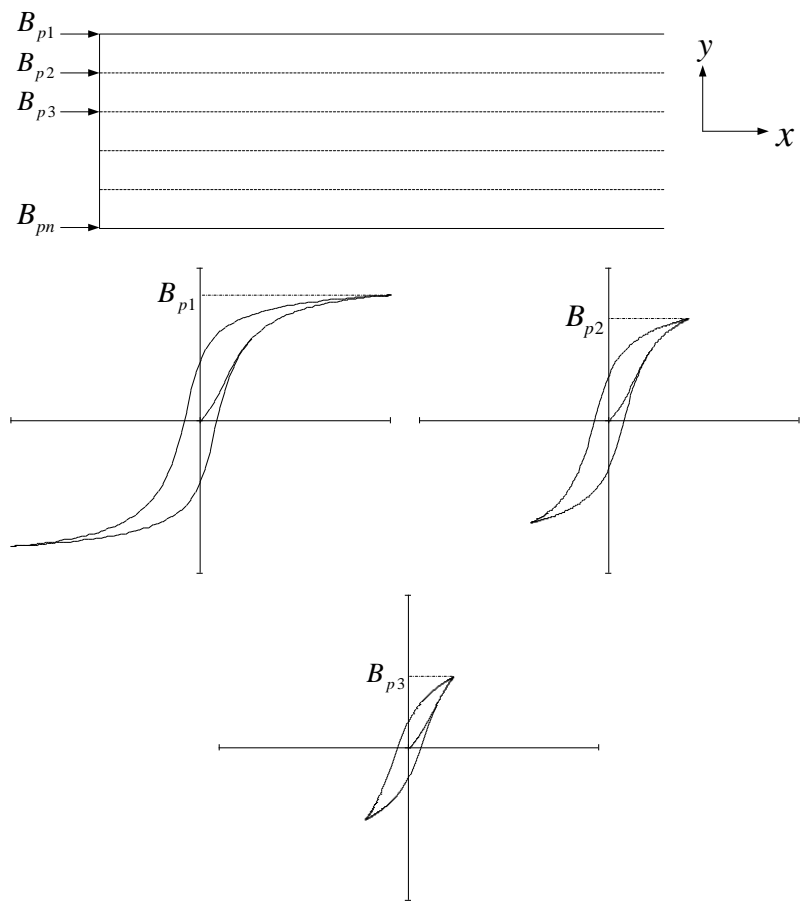

Fig. 3. Illustration of the variation of hysteresis loops across the lamination thickness. 


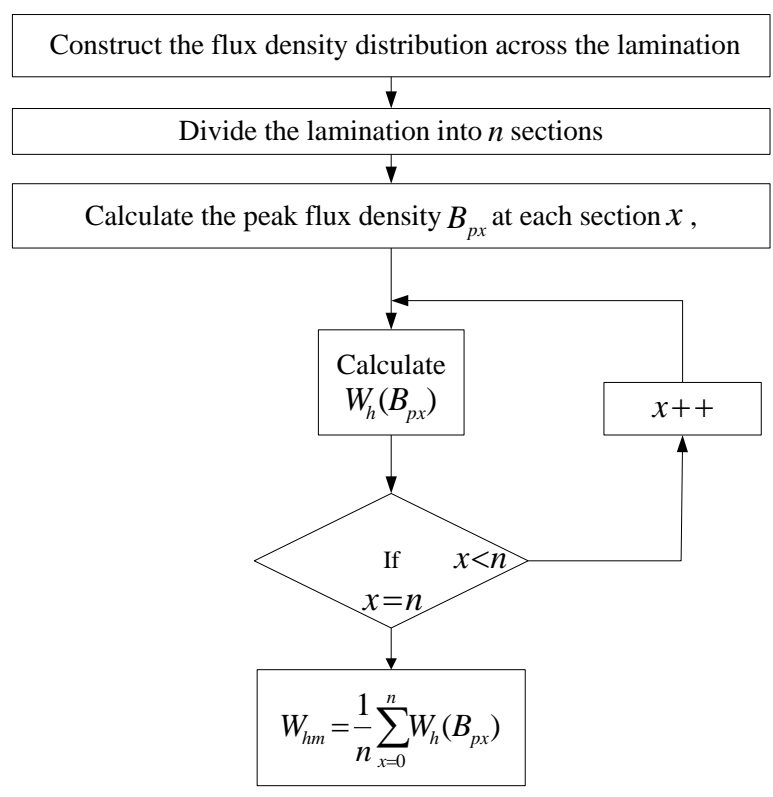

Fig. 4. Flow chart of the model.

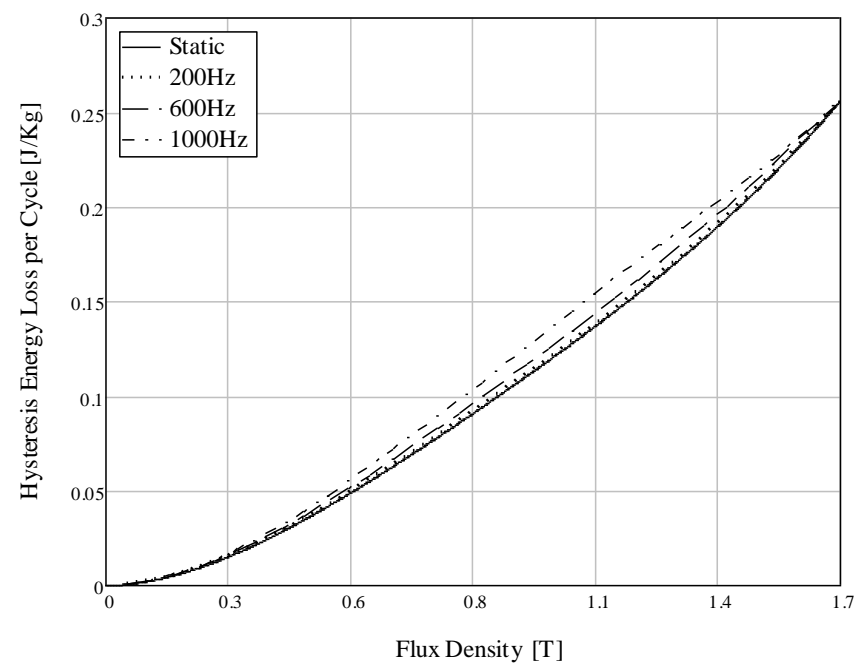

Fig. 5. Model result for un-annealed lamination.

TABLE II

METHODS OF HYSTERESIS LOSS SEPARATION

\begin{tabular}{ccccccc}
\hline \hline $\begin{array}{c}\text { Frequency } \\
{[\mathrm{Hz}]}\end{array}$ & $\begin{array}{c}\text { Flux } \\
{[\mathrm{T}]}\end{array}$ & $\begin{array}{c}\text { FE } \\
\text { Loss } \\
{[\mathrm{W} / \mathrm{kg}]}\end{array}$ & $\begin{array}{c}\text { Extrapo- } \\
\text { lation } \\
\text { Loss } \\
{[\mathrm{W} / \mathrm{kg}]}\end{array}$ & $\begin{array}{c}\% \\
\text { Error } \\
*\end{array}$ & $\begin{array}{c}\text { Model } \\
\text { Loss } \\
{[\mathrm{W} / \mathrm{kg}]}\end{array}$ & $\begin{array}{c}\% \\
\text { Error } \\
*\end{array}$ \\
\hline \multirow{2}{*}{50} & 1.00 & 6.03 & 6.02 & 0.18 & 6.00 & 0.41 \\
\cline { 2 - 7 } & 1.50 & 10.93 & 10.90 & 0.32 & 10.90 & 0.33 \\
\hline \multirow{2}{*}{200} & 1.00 & 24.60 & 24.07 & 2.18 & 24.16 & 1.79 \\
\cline { 2 - 7 } & 1.50 & 44.04 & 43.60 & 1.00 & 43.70 & 0.76 \\
\hline \multirow{2}{*}{600} & 1.00 & 76.35 & 72.20 & 5.45 & 75.98 & 0.49 \\
\cline { 2 - 7 } & 1.50 & 133.09 & 130.80 & 1.72 & 133.91 & -0.61 \\
\hline \multirow{2}{*}{2000} & 1.00 & 136.99 & 120.33 & 12.16 & 136.50 & 0.36 \\
\hline
\end{tabular}

* Based on the respective FE results

\section{RESULTS}

The finite section method described in section III can be employed by steel manufacturers to achieve accurate break down of core loss components at high frequency. This would help in the development of optimized steels for high frequency applications. The model can also be used to establish a data base of core loss components at different frequencies and flux densities. This database would be a first step of developing a formula that accounts for the variation of the hysteresis energy loss with the frequency. Such a formula can improve the prediction of core losses in high frequency transformers and high speed electrical machines, where the steel laminations are exposed to high frequency magnetic fields, and skin effect is pronounced. However, the mechanism of core losses in electrical machines is more complicated than single phase transformers, as the local magnetic field in some parts of the machine varies its direction in space [21]. Currently, no rotational loss data is available from the manufacturers and the designers are limited to alternating flux data, which accounts for the majority of the losses [22]. Here the improvement is in the separation of the alternating losses which can lead to better machine designs.

In this section, the developed model is used to separate the measured core losses in annealed and un-annealed laminations into hysteresis and eddy current loss. The separated losses are then used to analyze the effect of annealing on core loss components in laminations exposed to high frequency excitations.

\section{A. Testing Equipment}

The magnetic testing of the samples is carried out using a commercial test system. The system is capable of automatic core loss measurements using the Epstein frame, toroid tester and single sheet tester. The system is fully customized for high frequency testing with increased drive capabilities, and enhanced measurement capabilities, as it includes sample temperature monitoring used for testing above $1000 \mathrm{~Hz}$. Secondary waveform correction is used to keep the flux waveform sinusoidal during saturation. The experimental testing is performed using a 352-turn Epstein frame. The frame design allows testing at high frequencies without exceeding the system drive capabilities. The Epstein frame test has a test frequency range of $20 \mathrm{~Hz}-4000 \mathrm{~Hz}$. Core loss measurements at this range allow the gathering of core loss data not generally provided by steel manufacturers. In addition, the system is capable of measuring the AC hysteresis loops from 20 to $400 \mathrm{~Hz}$.

\section{B. Effect of annealing on the total core loss}

Comparative core loss measurements are performed on dry, wet annealed and un-annealed laminations with $0.61 \mathrm{~mm}$ thickness at various frequencies in the range of $20 \mathrm{~Hz}-$ $4000 \mathrm{~Hz}$. Fig. 6 shows that the particular annealing process (wet or dry) does not affect the total core loss measured at different frequencies. Table III shows a comparison of the measured core loss in the annealed and un-annealed samples at different frequencies. From Table III, it is clear that a significant reduction in the low frequency core loss is achieved by annealing. This reduction decreases with raising the frequency, until the effect of annealing on the total core 
TABLE III

CORE LOSS REDUCTION BY ANNEALING

\begin{tabular}{ccccc}
\hline \hline $\begin{array}{c}\text { Frequency } \\
{[\mathrm{Hz}]}\end{array}$ & $\begin{array}{c}\text { Flux Density } \\
{[\mathrm{T}]}\end{array}$ & $\begin{array}{c}\text { Un-annealed } \\
\text { Losses } \\
{[\mathrm{W} / \mathrm{Kg}]}\end{array}$ & $\begin{array}{c}\text { Annealed } \\
\text { Losses } \\
{[\mathrm{W} / \mathrm{Kg}]}\end{array}$ & \%Difference* \\
\hline 20 & 1 & 2.4531 & 0.5487 & 77.63 \\
50 & 1 & 6.734 & 1.9778 & 70.62 \\
60 & 1 & 8.4422 & 2.5647 & 69.62 \\
200 & 1 & 38.299 & 18.188 & 52.5 \\
600 & 1 & 193.3 & 130.14 & 32.67 \\
1000 & 1 & 424.11 & 323.54 & 23.71 \\
2000 & 1 & 1211.5 & 1104.9 & 8.80 \\
4000 & 0.85 & 2508.6 & 2424 & 3.37 \\
\hline
\end{tabular}

loss can be neglected at high frequencies and high densities, as shown in the measured core loss in Fig. 7. In addition, the magnetic properties of the material are recovered by annealing. This can be clearly observed in the measured magnetic permeability, shown in Fig. 8.

\section{Effect of annealing on the hysteresis Loss}

The reduction in the total core loss at low frequency achieved by annealing is mainly attributed to the reduction in the hysteresis loss component, shown in Fig. 9.The reduction in the hysteresis loss by annealing was also reported in $[13,14]$. The hysteresis loss is the dominant loss component at low frequencies. This can be seen from the ratio of hysteresis to eddy current at different frequencies in Fig. 10. At high frequency, the reduction in the hysteresis losses has less impact on high frequency core losses, as the eddy current loss forms the larger portion of the total core losses. Moreover, the hysteresis energy loss per cycle increases at high frequency due to skin effect, as illustrated in section III. Since the magnetic field distribution in steels with high permeability tends to be non-uniform, the hysteresis loss is more likely to be influenced by skin effect in the annealed samples.

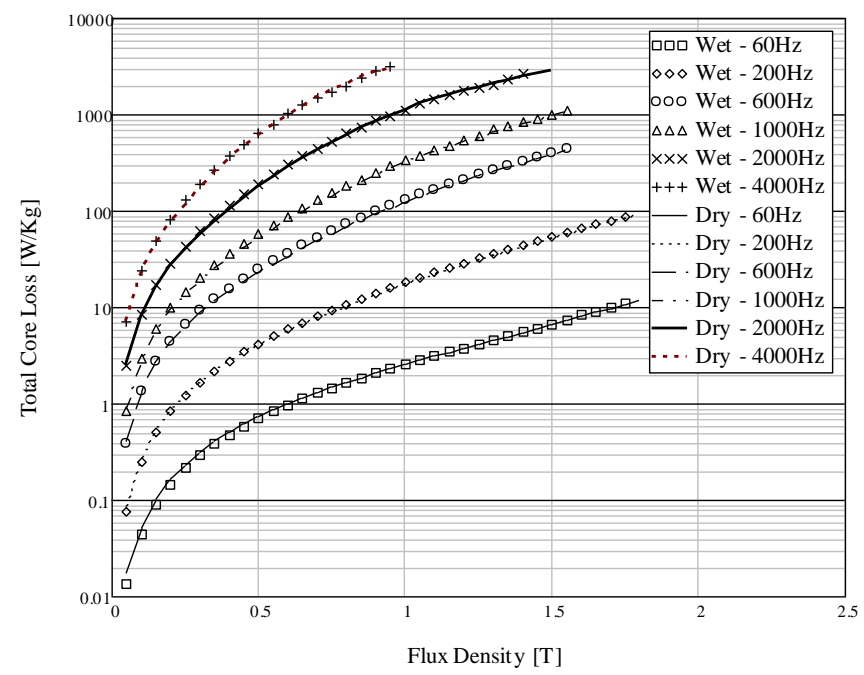

Fig. 6. Effect of the particular annealing process (wet or dy) on the measured total core loss.

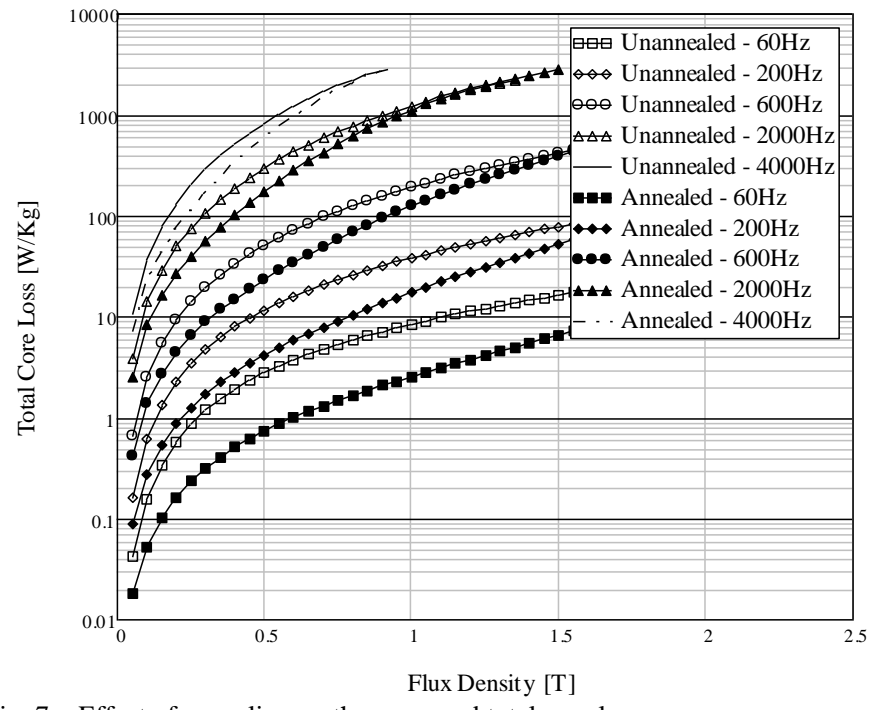

Fig. 7. Effect of annealing on the measured total core loss.

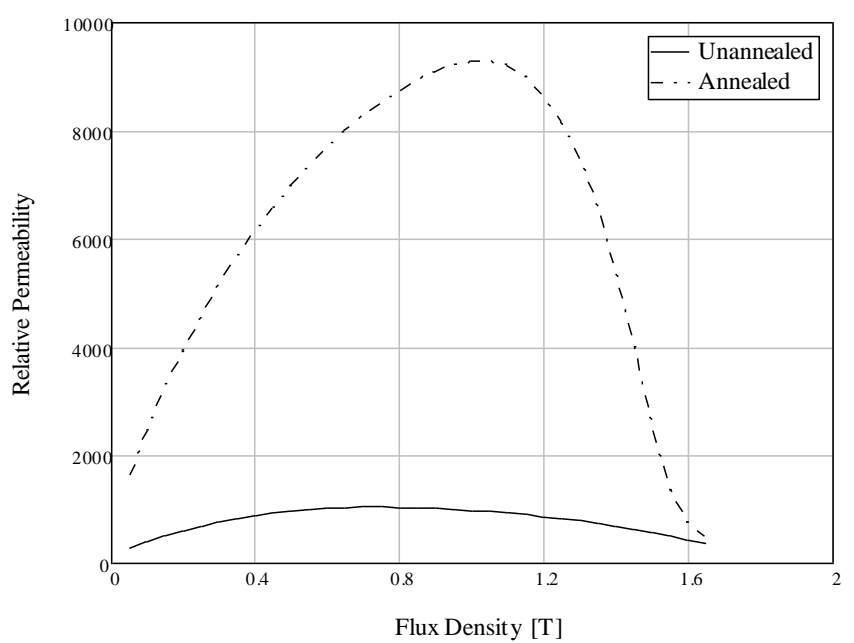

Fig. 8. Effect of annealing on the magnetic permeability measured at $20 \mathrm{~Hz}$.

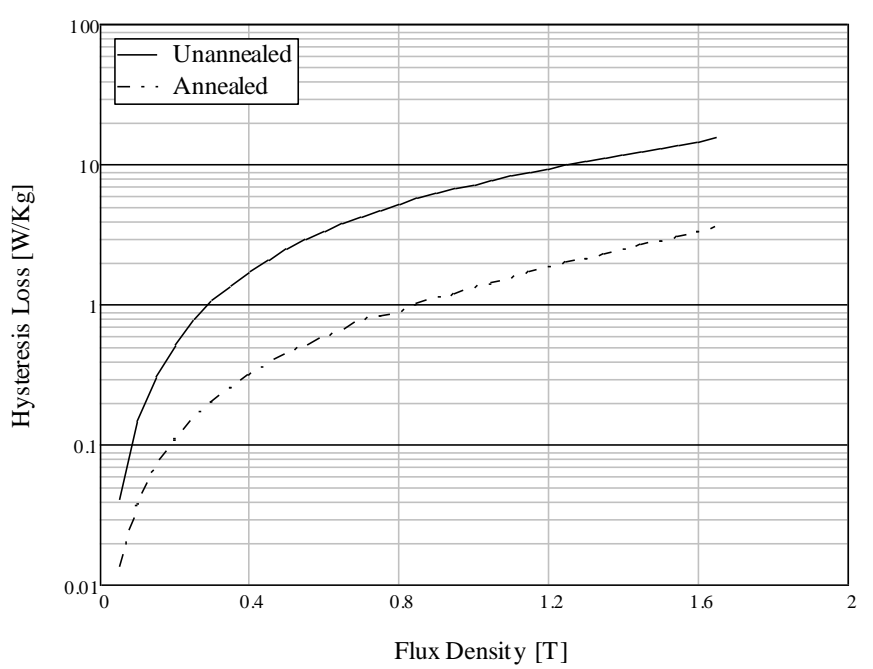

Fig. 9. Effect of annealing on the hysteresis loss at $60 \mathrm{~Hz}$. 


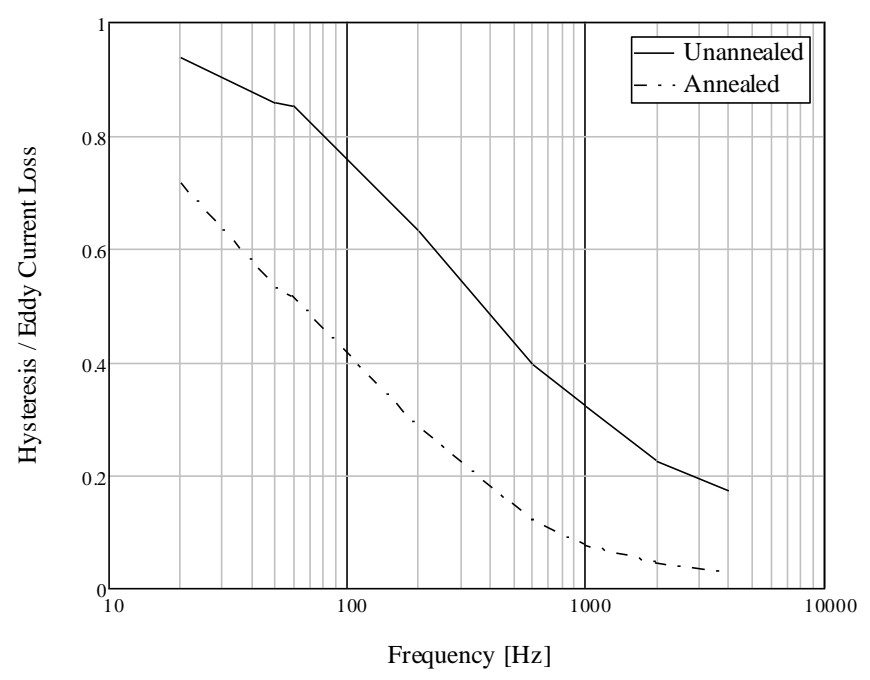

Fig. 10. Ratio of hystresis to eddy current losses at different frequencies.

\section{Effect of annealing on the eddy current loss}

While the annealing process is beneficial in terms of reducing the hysteresis loss and improving the magnetic properties of the material, it causes an increase in the eddy current loss. Fig. 11 shows the effect of annealing on eddy current loss at $1 \mathrm{~T}$ and $1.5 \mathrm{~T}$. It can be observed that the eddy current loss at $1 \mathrm{~T}$ is less affected by annealing for frequencies lower than $1000 \mathrm{~Hz}$. For higher frequencies, the eddy current loss is higher in the annealed samples. At 1.5T, the annealing increases the eddy current loss at all the tested frequencies. In order to study the effect of annealing on eddy current loss, the separated eddy current loss is compared with the results obtained from an analytical eddy current loss formula that accounts for the non-uniform flux density distribution inside the lamination. The eddy current losses at a certain frequency are calculated based on the flux penetration, which is determined by the dimensionless skin depth $\lambda$. The formula also takes into account the deviation between the flux density at the lamination boundary and the average flux density measured over the lamination. A full description of the mathematical development of the formula can be found in [7].

$P_{e}=\frac{\omega B_{p}^{2}}{2 \lambda \mu \rho} \frac{\left[\exp \left(\frac{4}{\lambda}\right)+2 \cos \left(\frac{2}{\lambda}\right) \exp \left(\frac{2}{\lambda}\right)+1\right]\left[\exp \left(\frac{4}{\lambda}\right)-2 \exp \left(\frac{2}{\lambda}\right) \sin \left(\frac{2}{\lambda}\right)-1\right]}{\left[1-2 \exp \left(\frac{4}{\lambda}\right) \cos \left(\frac{4}{\lambda}\right)+\exp \left(\frac{8}{\lambda}\right)\right]}$

As shown in Fig. 12, the formula predicts eddy current loss in the un-annealed sample accurately, while a significant deviation is found between the predicted and calculated eddy current loss in the annealed samples, where the eddy current loss is under estimated substantially. The formula is based on the solution of Maxwell's equations with an assumption of linear magnetic material. According to this assumption, the eddy current loss should be reduced in the annealed samples, where skin effect is more significant due to its high magnetic

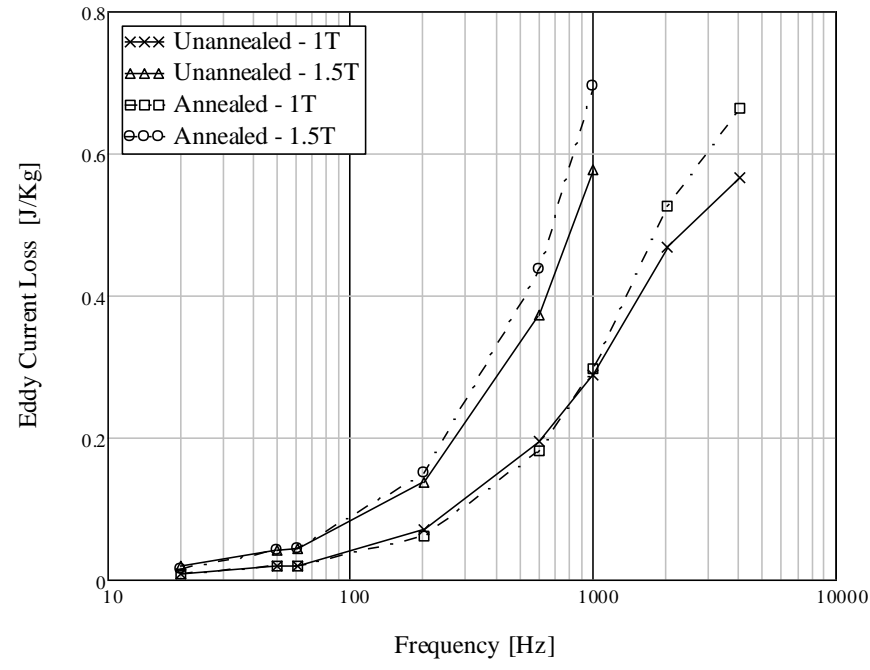

Fig. 11. Effect of annealing on eddy current loss.

permeability, as illustrated in Fig. 13, this forces the eddy currents to flow in a smaller area, increasing the resistance of the eddy current path and limiting the induced eddy currents, and thus reducing the eddy current losses as shown in the calculated loss in Fig. 14. In contrast, the measured eddy current loss in the annealed samples is much higher than the predicted values and even higher than the eddy current loss in the un-annealed samples.

The annealing process changes the magnetic properties of the material. This can be clearly seen in the measured hysteresis loops before and after annealing, shown in Fig. 15 and 16, respectively. Below the knee of the lower branch of the hysteresis curve for annealed samples, it is clear that a small change in the magnetic field causes a large increase in the flux density. While beyond the knee, the flux density variation is small corresponding to large changes in the magnetic field. This distinctive shape of the hysteresis loop in the annealed samples causes the local flux density to follow almost rectangular profiles $[15,16]$. The steep edges of the local flux density waveforms increase the eddy current loss in the annealed samples, especially at high flux densities, where the rectangular profiles of flux density are more pronounced. Moreover, the annealing process causes the grain size of the magnetic material to increase. Growing larger grains is associated with the existence of more widely spaced domain walls, which means that the individual walls have to move faster to produce certain flux change. The rapid movement of walls increases the eddy current loss as the eddy currents are located at the moving domain walls [17]. The effect of domain structure on eddy current loss can be neglected in the unannealed samples, as it has fine domain structure [18].

\section{CONCLUSION}

A model has been implemented to calculate high frequency hysteresis losses taking into account the non-uniform flux distribution. The results show that the assumption of constant hysteresis energy loss per cycle is only valid at low 


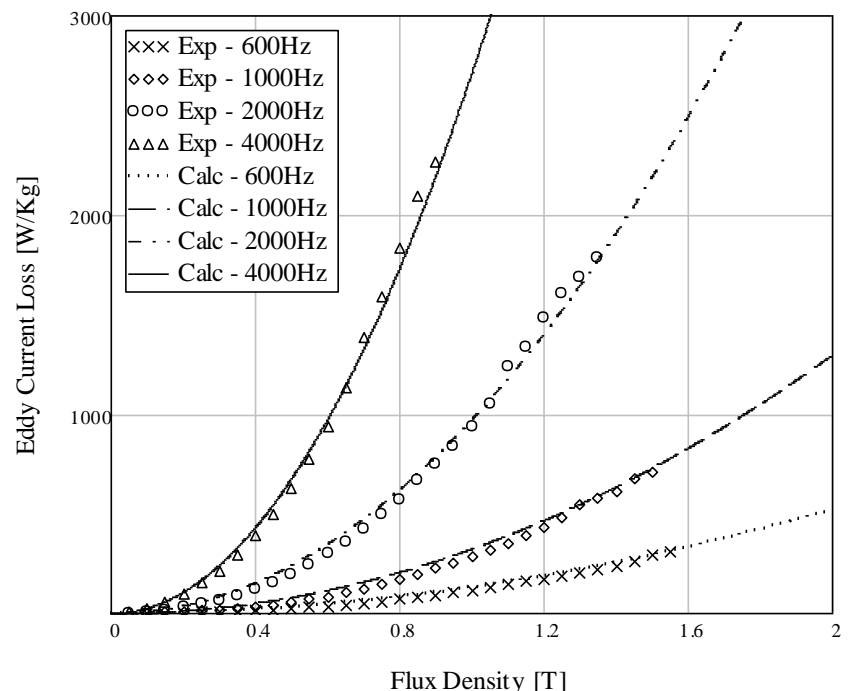

Fig. 12. Comparison between calculated and measured eddy current loss in the un-annealed laminations.

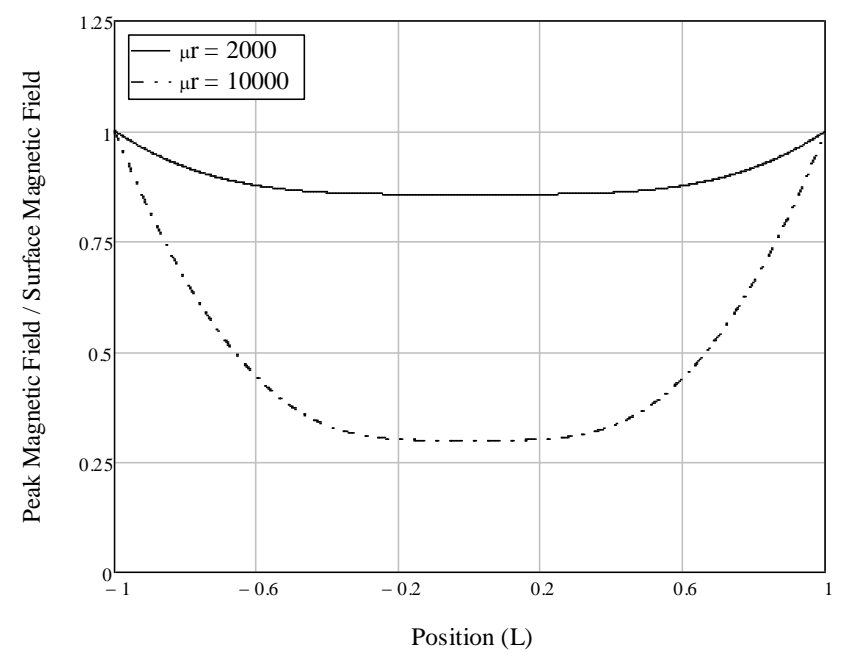

Fig. 13. Effect of the permeability on magnetic field distribution at $200 \mathrm{~Hz}$

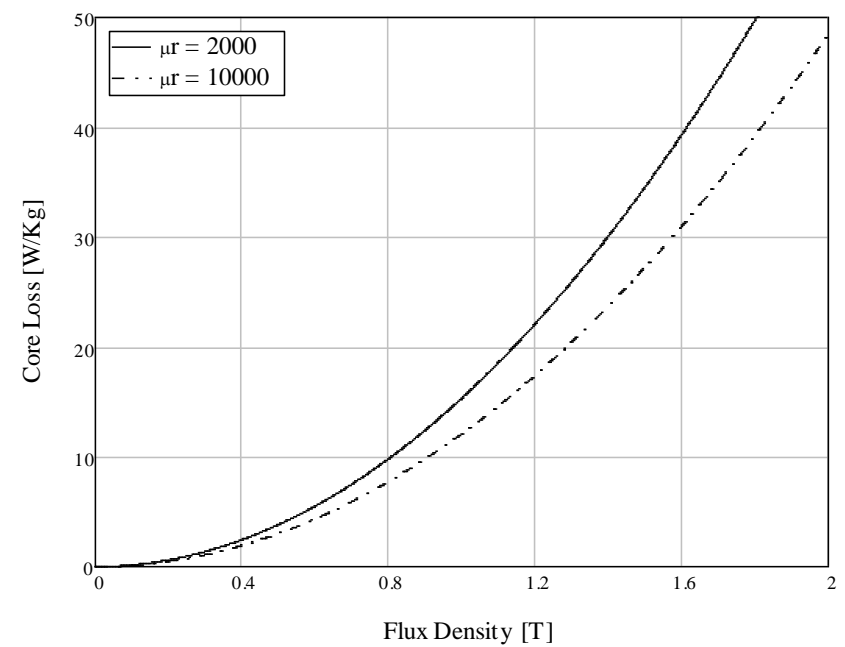

Fig. 14. Calculated eddy current loss for different values of magnetic permeability at $200 \mathrm{~Hz}$.

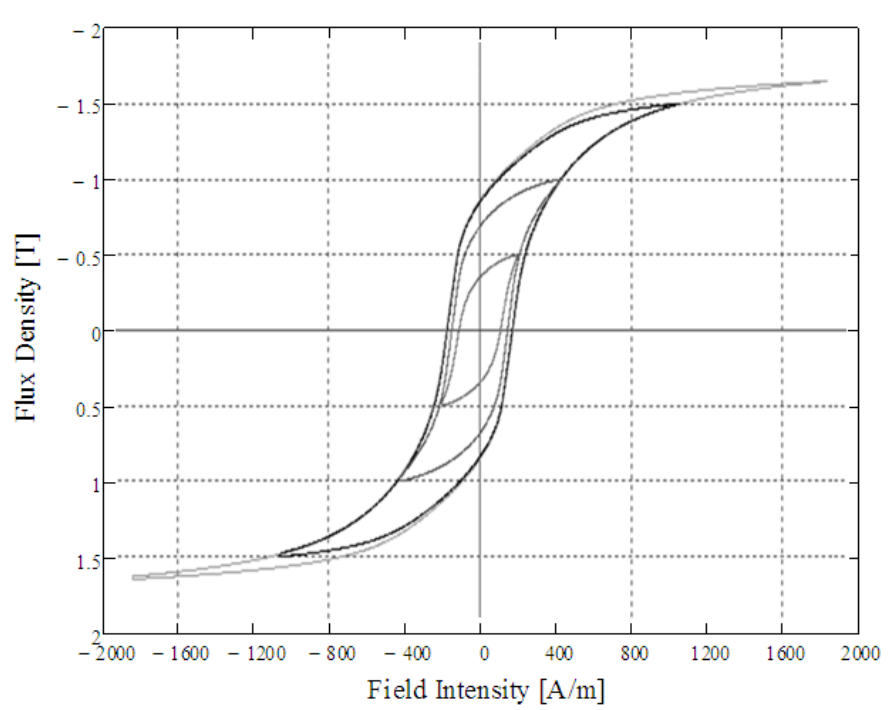

Fig. 15. Measured hysteresis loops in the un- annealed laminations at $20 \mathrm{~Hz}$.

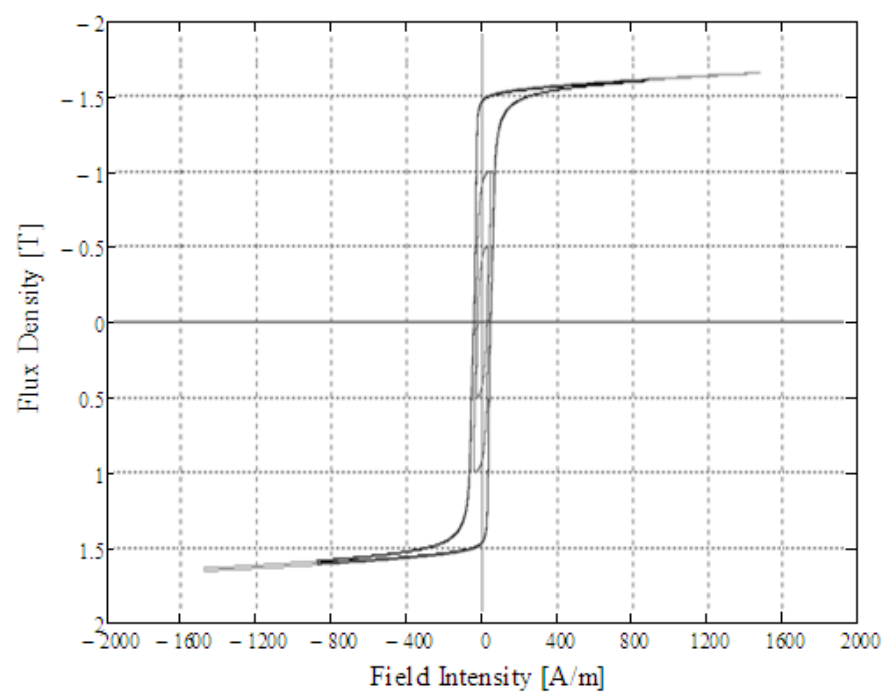

Fig. 16. Measured hysteresis loops in the annealed laminations at $20 \mathrm{~Hz}$.

frequencies where skin effect is negligible. While at high frequency, the flux density amplitude varies at each point inside the lamination. As a result, the hysteresis energy loss per cycle increases, and tends to change linearly related to the flux density.

Comparative high frequency core loss measurements have been performed on annealed and un-annealed samples at various frequencies up to $4000 \mathrm{~Hz}$. Accurate separation of core loss components is achieved by using the model to calculate the hysteresis losses taking into account the realistic flux distribution. The annealing process has been found to significantly decrease the hysteresis losses. On the contrary, the eddy current losses in the annealed samples are higher than the un-annealed samples, especially at high frequencies and high flux densities. The eddy current losses have been estimated using a formula that accounts for the effect of magnetic field distribution on the eddy current loss. The formula predicts eddy current loss accurately in the unannealed samples for frequencies up to $4000 \mathrm{~Hz}$. However, it 
underestimates eddy current losses in the annealed samples, where the local flux density waveforms inside the lamination are almost rectangular. These rectangular flux profiles are attributed to the special shape of the hysteresis loops in the annealed samples.

\section{ACKNOWLEDGMENT}

This project is part of the R\&D program of the NSERC Chair entitled "Energy efficiency in electrical machines for small renewable energy production systems" established in 2009 at Concordia University. The authors acknowledge the support of the Natural Sciences \& Engineering Research Council of Canada and Hydro-Québec.

\section{REFERENCES}

[1] Gilbert A. McCo and et al, Energy-Efficient Electric Motor Selection Handbook, Revision 3, January 1993.

[2] C. P. Steinmetz, "On the law of hysteresis (originally published in 1892)," Proc. IEEE, vol. 72, no. 2, pp. 196-221, Feb. 1984

[3] G.Bertotti, "General properties of power losses in soft ferromagnetic materials,” IEEE Trans. Magn. Vol. 24, pp. 621-630, January 1988.

[4] Y. Chen and P. Pillay, "An improved formula for lamination core loss calculations in machines operating with high frequency and high flu density excitation," IEEE 37th IAS Annual Meeting, vol. 2, pp. 759766, 13-18 Oct 2002.

[5] D. M. Ionel, M. Popescu, S. J. Dellinger, et al., "on the variation with flux and frequency of core loss coefficitens in electrical machine", IEEE Trans. Magn Vol. 42, No. 3, pp. 658-666, May/June 2006.

[6] Y. Zhang, M.-C Cheng, P. Pillay, "Magnetic Characteristics and Excess Eddy Current Losses" in proc. IEEE Industry Applications Society Annual Meeting, 2009., pp. 1-5.

[7] Yu Zhang, Rui Guan, Pillay, P. and Cheng, M.-C. "General core loss models on a magnetic lamination" in proc. IEEE Int. Electric Machines and Drives Conf. , 3-6 May 2009, pp. 1529 - 1534.

[8] A. Boglietti, A. Cavagnino, "Iron Loss Prediction with PWM Supply: An Overview of Proposed Methods from an Engineering Application Point of View", in proc. IEEE Int. Industry Applications Society Annual Meeting, 2007, pp. $81-88$.

[9] B. D. Cullity and C. D. Graham, "Introduction To Magnetic Materials", 2nd ed. John Wiley \& Sons, 2009.

[10] Y. Zhang, M-C. Cheng, P. Pillay and B. Helenbrook, " High order finite element model for core loss assessment in a hysteresis magnetic lamination", J. Appl. Phys. 106, 43911 (2009).

[11] H. Hauser, "Energetic model of ferromagnetic hysteresis: Isotropic magnetization", J. Appl. Phys. 96, 2753 (2004).

[12] T.L.Mthombeni and P.Pillay, "Physical Basis for the Variation of Lamination Core Loss Coefficients as a Function of Frequency and Flux Density." IECON, France, 2006.

[13] A. Boglietti, A. Cavagnino, M. Lazzari, and M. Pastorelli, "The annealing influence onto magnetic and energetic properties in soft magnetic material after punching process," in Proc. IEEE Int. Electric Machines and Drives Conf., vol. 1, June 1-4, 2003, pp. 503-508.

[14] L. T. Mthombeni, and P. Pillay, "Core Losses in Motor Laminations Exposed to High-Frequency or Nonsinusoidal Excitation," IEEE Trans. On Ind. App., Vol. 40, No. 5, Sep. /Oct. 2004.

[15] I. Mayergoyz and C. Serpico, "Nonlinear diffusion of electromagnetic fields and excess eddy current losses" J. Appl. Phys. 85, 4910 (1999).

[16] C. Serpico, C. Visone, I. Mayergoyz et al., "Eddy current losses in ferromagnetic laminations" J. Appl. Phys. 87, 6923 (2000).

[17] R. H. Pry and C. P. Bean, "Calculation of the energy loss in magnetic sheet materials using a domain model," J. Appl. Phys., vol. 29, pp. 532 533, Mar. 1958.

[18] P. Beckley, Electrical Steels: A Handbook for Producers and Users. Newport, U.K.: European Electrical Steels, 2000.

[19] J. Gyselinck, L. Vandevelde, J. Melkebeek, et al., "Calculation of eddy currents and associated losses in electrical steel laminations," IEEE Trans. Magn., vol. 35, no. 3, pp. 1191 -1194, May 1999.

[20] J. Pippuri, A. Belahcen, E. Dlala, and A. Arkkio, "Inclusion of Eddy Currents in Laminations in Two-Dimensional Finite Element
Analysis"," IEEE Trans. Magn., vol. 46, no.8, pp. 2915 -2918, Aug. 2010.

[21] Y. Guo, J. Zhu, J. Zhong et al., "Measurement and Modeling of Rotational Core Losses of Soft Magnetic Materials Used in Electrical Machines - A Review", IEEE Trans. Magn., vol. 44, no. 2, pp. 279291, Feb. 2008.

[22] N. Alatawneh and P. Pillay, "Design of a novel test fixture to measure rotational core losses in machine laminations," in proc. IEEE Energy Conversion Congress and Exposition Conf. 2011, 17-22 Sept. 2011, pp. 422-440.

Maged Ibrahim (S'10) received the Bachelor's degree in 2008 from Alexandria University, Alexandria, Egyp, and the M.S. degree in 2011 from Concordia University, Montreal, Canada, where he is currently pursuing the $\mathrm{Ph} . \mathrm{D}$. degree in electrical engineering.

His research interests include core losses in magnetic materials, electrical machines and power electronics.

Pragasen Pillay (F'05) received the Bachelor's degree from the University of Kwa-Zulu Natal, Durban, South Africa, in 1981, the Master's degree from the University of Kwa-Zulu, Natal, Durban, South Africa, in 1983, and the Ph.D. degree from Virginia Polytechnic Institute and State University, Blacksburg, in 1987.

Currently, he is a Professor in the Department of Electrical and Computer Engineering, Concordia University, Montreal, Canada, where he holds the NSERC/Hydro Quebec Industrial Research Chair. From 1988 to 1990, he was with the University of Newcastle-upon-Tyne, Newcastle-upon-Tyne, U.K. From 1990 to 1995, he was with the University of New Orleans. From 1995 to 2007 he was with Clarkson University, Potsdam, NY, where he held the Jean Newell Distinguished Professorship in Engineering. He is also an Adjunct Professor at the University of Cape Town, Cape Town, South Africa. His research and teaching interests are in modeling, design, and control of electric motors and drives for industrial and alternate energy applications.

Dr. Pillay is a member of the IEEE Power Engineering, IEEE Industry Applications (IAS), IEEE Industrial Electronics, and IEEE Power Electronics Societies. He is a member of the Electric Machines Committee and Past Chairman of the IEEE Industrial Drives Committee of the IAS, Past Chairman of the Induction Machinery Subcommittee of the IEEE Power Engineering Society, Past Chairman of the Awards Committee of the IAS Industrial Power Conversion Department. He has organized and taught short courses in electric drives at IAS Annual Meetings. He is a Fellow of the Institution of Electrical Engineers and Technologists, U.K., and a Chartered Electrical Engineer in the U.K. He is also a Member of the Academy of Science of South Africa. He was a recipient of a Fulbright Scholarship for his Ph.D and received the Order of Mapungubwe from the President of South Africa in 2008 for contributions to South Africa in the area of energy conservation. 